being completely gone in three months. The drooping of the face became less noticeable, but did not disappear. She remained well about three months, when her old symptoms reappeared. She noticed now that in certain places her sense of touch was not as acute on the right as on the left side. The facial pains ceased after an attack of epistaxis, but the other symptoms persisted. In this condition she consulted the author, who noted movements in the fingers, hand, forearm, toes, foot, and leg of the right side. The movements were slow, rhythmical, unceasing, involuntary, and yet, as it were, purposive, and they ceased during sleep. There was facial hypokinesis on the right side. The cutaneous sensibility was somewhat diminished in the parts in which the movements occurred. But there was no loss of power in the right extremities; the eleotrio irritability was unchanged; the reflex excitability, including the phenomenon of tendon-reflex, was normal, and the circumference of the limbs was the same on both sides. Bromide of potassium was given in increasing doses, and the continuous current, from the cervical and lumbar regions of the cord 'downwards to the affected muscles, was applied every other day for about ten minutes. In a few monthe the patient had completely recovered.

The important points in this case are (1) the fact that the disease occurred in an individual who had previously enjoyed good health; (2) the partial recovery and subsequent relapse; and (3) the ultimate complete recovery of the patient. The case is one of idiopathic or primary athetosis, as distinguished from secondary or symptomatio athetosis, in which we hare a history of hemiplegia, epilepsy, brain atrophy, \&c. Of the primary disease only five cases are on record, and Gnauck gives a comparative analysis of them. In the only instance in which an autopsy was made, a focus of softening was found in the 'corpus striatum and lenticular nucleus' of one side. In the case just reported there was probably an affection of the outer part of the left half of the pons. The author distinguishes between symptomatic athetosis and cases of athetoid. movements: in the former, the symptoms very closely resemble those of primary athetosis, in the latter, they differ from these in some important respects.

$$
\text { W. J. Dodds, M.D., D.Sc. }
$$

\title{
A Case confirming Cerebral Localisation. Dr. Tamburim
} (Rivista Sperimentale di Freniatria, Anno V. Fascicolo III.) gives an account of an imbecile, 45 years old, bearing the honoured name of Paul Veroneso. He was subjoct to epileptic fits, which were often 
followed by delirium. During an attack of this kind he killed an idiot with a knife. The epilepsy dated from infancy, and was accompanied by atrophy and paralysis of the left arm and leg. He was four years and a half in the asylum at Reggio, where be died. The fits had become so frequent that they could not be counted. The attacks were always preceded by an aura; then followed clonio movements, which began in the paralysed and wasted left arm. Sometimes the convulsions were limited to the arm, and extended to the face and leg of left side; sometimes they involved the whole body. The face was less developed on the left side than on the right. In fact the whole left side was smaller than the right; still the leg was not so much paralysed as the arm. The man's intelligence was very deficient, the power of speech limited, and the command of the vocal apparatus imperfect. Words were pronounced with great effort and with repetition and stuttering.

The left hemisphere of the brain was found to be normal, it weighed 510 grammes, but the right hemisphere was much smaller, weighing only 260 grammes; the whole brain weighed 930 . On the right side, the ascending parietal convolution (the posterior median of Ecker) was atrophied and indurated, especially at its external part. This atrophy and induration involved the neighbouring portion of the ascending frontal gyrus anterior median of Ecker and the lower parts of the second and third frontal gyri. Behind this circle of sclerosed matter there was a cavity about the size of a pigeon's egg, full of purulent matter, extending through the left temporal lobe and occupying the place of the island of Reil, all vestiges of which had disappeared. The right thalamus opticus, crus cerebri, and surface of the pons on the right side were also found diminished in size and hardened in texture. The spinal cord was found atrophied, especially on the cervico-dorsal region. The sclerosed part examined by microscope showed an increase in the connective tissues and in the gyri round the fissure of Sylvius; amyloid corpuscles and white cells were found around the vessels.

Tamburini remarks that the atrophy of the left hemisphere contiuned into the crus cerebri, the pons and anterior pyrumid of the same side, and then passing into the half of the spinal cord taken into connection with the atrophy and paralysis of the left side of the body, furnishes a striking proof of the dependence of the trophic and motor funotions, not only in the cord and pons, but also in the cerebral hemisphere as well as of the crossing of the nervous tract denied by Brown-S6́quard. 
The loss of power in the left arm and leg and in the face is explained by the injury to the external portions of the ascending parietal, the ascending frontal, and the lower part of the third frontal.

The dissection of the brain, writes the learned physiologist, signally confirms the criterion for diagnosis brought to light by Hughlings-Jackson and experimentally confirmed by us (Tamburini and Luciani) that the lesion of the motor centre of the cortex cerebri may be diagnosed from the muscles first affected by the epileptic attack. The injury to speech is explained by the destruction of the island of Reil, which, in this case be it noted, was on the right side of the brain.

In considering the atrophy and sclerosis of the right optic thalamus, Tamburini enumerates the views of some distinguished neurologists on the function of this large basal ganglion. Luys holds that it contains four special centres for the representation of smell, sight, hearing and general sensibility. Ferrier thinks it to be a ganglion of interruption or centre of convergence of the sensory tracts. Fournier, Carville, Duret and Crichton-Browne hold that it is a centre of general sensibility. Nothnagel found that destruction of the optic thalamus was followed by a loss of sensibility and also of voluntary motion. Meynert, on the other hand, holds that the sensory impressions coming from the periphery are transformed in the optic thalami into movements; they are thus the automatic centres of reflex unconscious motions. Schiff, Lussana and Lemoigne concluded from their experiments that the optic thalami are centres for the motions of the arm and hand of the opposite side. The fact that in this case the atrophy of the optic thalamus was a continuation of the affection of the motor gyri, induces Dr. Tamburini to think that the thalamus is a conductor of the motor fibres, especially of the upper limb.

Hardening of the hippocampus major was found in this case; analysing the observations of five pathologists, he shows that it was found in 60 epileptics out of 272 dissections. He is of opinion that this lesion is only found in old cases of epilepsy. According to Meynert, the cornu ammonis contains peculiar pyramidal cells, but the destruction of the hippocampus causes no loss of any motor powers, nor does its removal bring any modification in epileptic convulsions. Tamburini recalls the views of Ferrier, who places the seat of olfactory sensation in the subiculum of the hippocampus. For these reasons he concludes that the hardening of 
this portion of brain has no causal influence on the production of epilepsy. He considers that the morbid process, so well and learnedly described, commenced in the cortex cerebri, and then descended through the basal ganglia to the spinal cord.

Caizergues' Case of Cerebral Localisation.-Dr. Caizergues, in a pamphlet of eleven pages, which first appeared in the 'Montpellier Médical,' describes a case of cerebral hæmorrhage, in which the new views as to the functions of the cortex cerebri and white matter of the centrum ovale are well illustrated. The man, 38 years of age, was admitted into the hospital at Montpellier with mitral contraction and insufficiency and contraction of the aorta. The arteries were believed to be atheromatous. Suffering from diarrhœe he rose from his bed, and returned exclaiming, "I cannot move my fist." Then a feeling of tingling, similar to what he had felt in the arm, appeared in the right leg, and ten minutes later the whole right side was paralysed. He was heard to say to the religieuse, "Ma mère," and these were the last intelligible words he was heard to speak, though he tried to make himself understood by signs. The interne ascertained the presence of hemianessthesia nicely limited to the right side of the body. There was no trembling or convulsions. He died about three hours after the first attack. An effusion of blood was found in the left hemisphere, which stretched in an oblique direction from above downwards and from behind forwards. The blood had raised the convolutions of the motor zone without injuring their tissue, and had gained the surface at one point near the union of the middle and upper third of the ascending parietal (posterior median of Ecker). The mass of the clot, about the size of a small apple, extended obliquely from below the fissure of Rolando to the fissure of Sylvius. Neither the nucleus lenticularis nor the nucleus caudatus was touched, but the internal capsule which separates them was found destroyed at its posterior and exterual part. The optic thalamus had escuped injury.

Dr. Caizergues thinks that the blood escaped gradually from the giving way of several miliary aneurisms. He holds that the paralysis of the arm and then of the leg was due to the destruction of the conducting white fibres of the centrum ovale, and not to any lesion of the motor area of grey matter, which Dr. Caizergues regards as left uninjured, although the blood forced its way to the surface at one point. The hemianesthesia is explained by the destruction of the internal capsule of the corpus striatum, and 
the aphasia is explained by the rupture of the lower pediculofrontal fibres.

William W. Ireland.

Lactic Acid as a Hypnotic.-Dr. Maragliano has recorded in the Rivista Sperimentale di Freniatria (Anno V. Fascicolo III.) the result of some experiments upon the hypnotic and sedative properties of laotic acid upon the insane. He mentions two theories upon the production of sleep. Some physiologists have advanced that under the influence of fatigue oxygen disappears from the blood, causing inactivity of the nervous centres and sleep, but the provision .of oxygen being renewed, their diminished powers are restored, and the nan awakes. Others hold that, though the quantity of oxygen in the blood is not less during sleep than during the waking state, it is quickly absorbed by those materials, such as creatine and lactic acid, which are the result of the disintegrating activity of the bodily organism, and which require to be oxidised ere they are eliminated. When this oxidisation has been accomplished, the brain can again make use of the oxygen in the blood and resume its proper functions.

According to the experiments of Ranke and Preyer, the artificial introduction of lactic acid and other products of retrograde metamorphosis are capable of producing weariness and sleep. With this theory in mind a number of German physicians used lactate of soda or lactic acid in the treatment of sleeplessness, but the results were somewhat disappointing. Doctors Maragliano and Sepilli tried these drugs in about a hundred cases of insanity. They found that if given (lactic acid in doses of 8 to 10 grammes and lactate of soda 12 to 15 grammes) three or four hours before bedtime, they were sufficient to subdue the insomnia of quiet melancholia; but they had little or no effect if given immediately before going to bed. In more decided cases of agitation and sleeplessness they were found very inferior to chloral and morphia, besides being too costly for ordinary use. They were also apt to produce nausea and vomiting.

W. W. IreLANd.

Relations of Brain, Mind, and Higher Nerve Function.In an able address delivered at the beginning of the session of the Pathological and Clinical Society of Glasgow, Dr. Alexander Robertson disousses some of the pathological and physiological relations of brain, mind, and higher nerve function. He begins by 